\title{
An Unusual case of Tolosa hunt syndrome with cerebral involvement Presented as optic perineuritis
}

\section{Nirna Hazeera Zahar ${ }^{1, *}$, Norlaila Talib ${ }^{1}$, Lott Pooi Wah ${ }^{2}$}

${ }^{1}$ Ophthalmology Hospital Serdang, Selangor, MYS

${ }^{2}$ Ophthalmology, University of Malaya Medical Centre, Kuala Lumpur, MYS

*Corresponding Author: Nirna Hazeera Zahar, Ophthalmology Hospital Serdang, Selangor, MYS

Received date: 13 June 2021; Accepted date: 21 June 2021; Published date: 23 June 2021

Citation: Zahar NH, Talib N, Wah LP. An Unusual case of Tolosa hunt syndrome with cerebral involvement Presented as optic perineuritis. J Med Case Rep Case Series 2(6): https://doi.org/10.38207/jmcrcs20210067

Copyright: (C) 2021 Nirna Hazeera Zahar. This is an open-access article distributed under the terms of the Creative Commons Attribution License, which permits unrestricted use, distribution, and reproduction in any medium, provided the original author and source are credited.

\begin{abstract}
:
Purpose: To report an unusual case of Tolosa Hunt Syndrome (THS) with cerebral involvement and initial presentation of optic perineuritis in a child. Method: A retrospective case report

Results: A 15-year-old female previously healthy presented with sudden onset blurry vision left eye progressively worsening over 5 days, headache and vomiting. Visual acuity of the left eye was CF with positive relative afferent pupillary defect (RAPD). Optic nerve function tests were impaired with loss of red desaturation and light brightness. EOM full and no other cranial nerves involvement. Fundus examination of the left eye revealed a normal optic disc with CDR 0.3, normal macula, and retina. Blood parameters were normal. Urgent CECT brain revealed left optic nerve is thickened measuring $0.5 \mathrm{~cm}$ (width) compared to the right optic nerve, which measures $0.3 \mathrm{~cm}$ with minimal streakiness seen surrounding the left optic nerve. MRI noted the presence of inflammatory mass at the region of the left cavernous sinus extending till the orbital apex with acute infarctions of the right putamen and body of the right caudate nucleus. She was referred to neuro medical and investigated for APLS and THS. Subsequently, her vision improved spontaneously. However, a week, later on, follow-up, noted the presence of 3rd and 4th cranial nerve involvement, MRI was repeated and presence of 'tram track' lesion over the optic nerve. She was started on iv methylprednisolone and her condition improved.

Conclusion: THS with cerebral involvement and optic perineuritis is a rare entity. Contrasted MRI is a diagnostic modality of choice. The early course of steroid commencement may hasten the recovery as well as prevent progression and complication.
\end{abstract}

\section{Introduction}

Tolosa Hunt syndrome is a rare syndrome caused by nonspecific granulomatous inflammation of the cavernous sinus or superior orbital fissure. It is characterized by unilateral orbital or periorbital headaches, along with ophthalmoplegia involving $3^{\text {rd }}, 4^{\text {th }}$, and $6^{\text {th }}$ cranial nerves. It may also involve the sympathetic fibers of the internal carotid artery within the cavernous sinus and parasympathetic fibers of the oculomotor nerve. Tolosa Hunt syndrome was first described in 1954 by Dr. Eduardo Tolosa, a Spanish neurosurgeon [1]. Followed by Hunt et al. in 1961. Smith and Taxdal called it Tolosa Hunt Syndrome for the first time in 1966 [2]. The annual estimated incidence of THS is about one case per million per year [3] Diagnosis of TSH usually assisted by imaging such as MRI/MRA which provides detail regarding inflammation of the cavernous sinus. Biopsy also may help in diagnosis but rarely done due to difficult

\section{Case report}

A 15 years old female previously healthy presented with sudden onset blurry vision left eye progressively worsening over 5 days, associated with headache and vomiting. Visual acuity of the left eye was CF with positive relative afferent pupillary defect (RAPD). Optic nerve function tests were impaired with loss of red desaturation and light brightness. EOM was full and no other cranial nerves involvement. procedures.

Optic peri neuritis is an uncommon variant of idiopathic orbital inflammatory disorder, involving the optic nerve sheath and surrounding tissues [4]. Optic peri neuritis may be mistaken with optic neuritis with both having similar presentation such as visual loss, pain on movement, and normal or swollen disc. However, the responses to treatments are different so it is important to distinguish between these two, mainly by radiographic changes and clinical features.

Tolosa hunt syndrome associated with optic peri neuritis is a rare condition. Both can be idiopathic and cause inflammation and the treatment is usually steroid. We report an unusual case of Tolosa Hunt Syndrome (THS) with cerebral involvement with initial presentation of optic perineuritis in a child.

Fundus examination of the left eye revealed a normal optic disc with CDR 0.3, normal macula, and retina. Blood parameters were normal. Urgent CECT brain revealed left optic nerve is thickened measuring $0.5 \mathrm{~cm}$ (width) compared to right optic nerve measures $0.3 \mathrm{~cm}$ with minimal streakiness seen surrounding the left optic nerve. MRI noted the presence of inflammatory mass at the region of the left cavernous 
sinus extending till the orbital apex with acute infarctions of the right putamen and body of the right caudate nucleus.

She was referred to neuromedical and investigated for APLS and THS. She underwent extensive workup to rule out autoimmune and infective conditions however no significant findings noted except her blood was slightly anemic. Subsequently, her vision improves spontaneously. She was discharged with the vision on 6/18.

However, a week, later on, follow-up, noted the presence of $3 \mathrm{rd}$ and 4th cranial nerve involvement with complete ptosis left eye. MRI was repeated and noted the presence of 'tram track 'lesion over the optic nerve. She was started on iv methylprednisolone $500 \mathrm{mg}$ od for 5 days and oral prednisolone on tapering doses. Her condition improved dramatically after the administration of steroids.

After 2 months completed steroid therapy, this patient came back with left eye partial ptosis and limited EOM involving 4th cranial nerve. The vision was 6/9 with no RAPD. She was given another cycle of steroid therapy and symptoms improved.

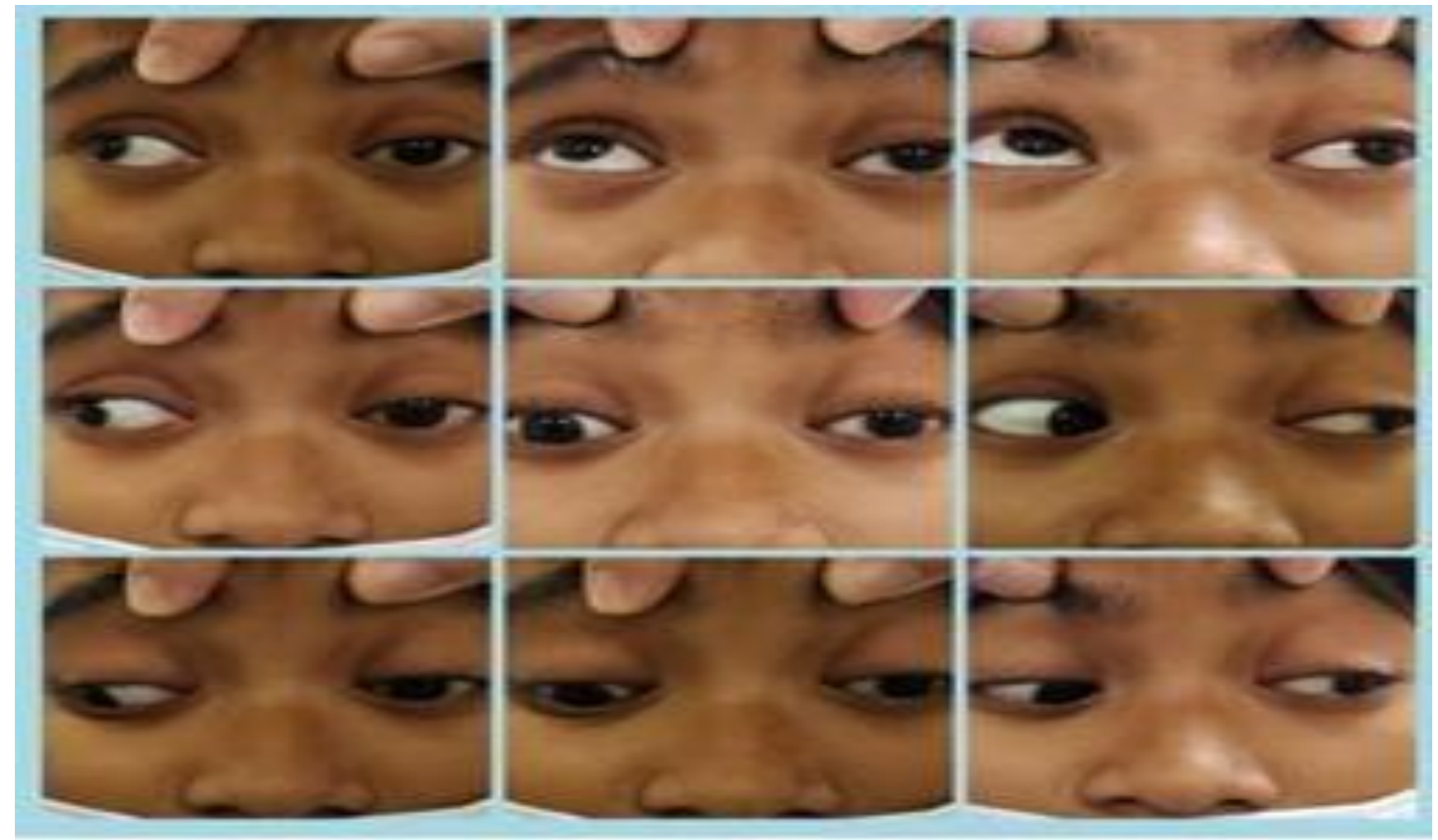

Figure 1: Restriction of left extraocular muscle movement with left eye ptosis

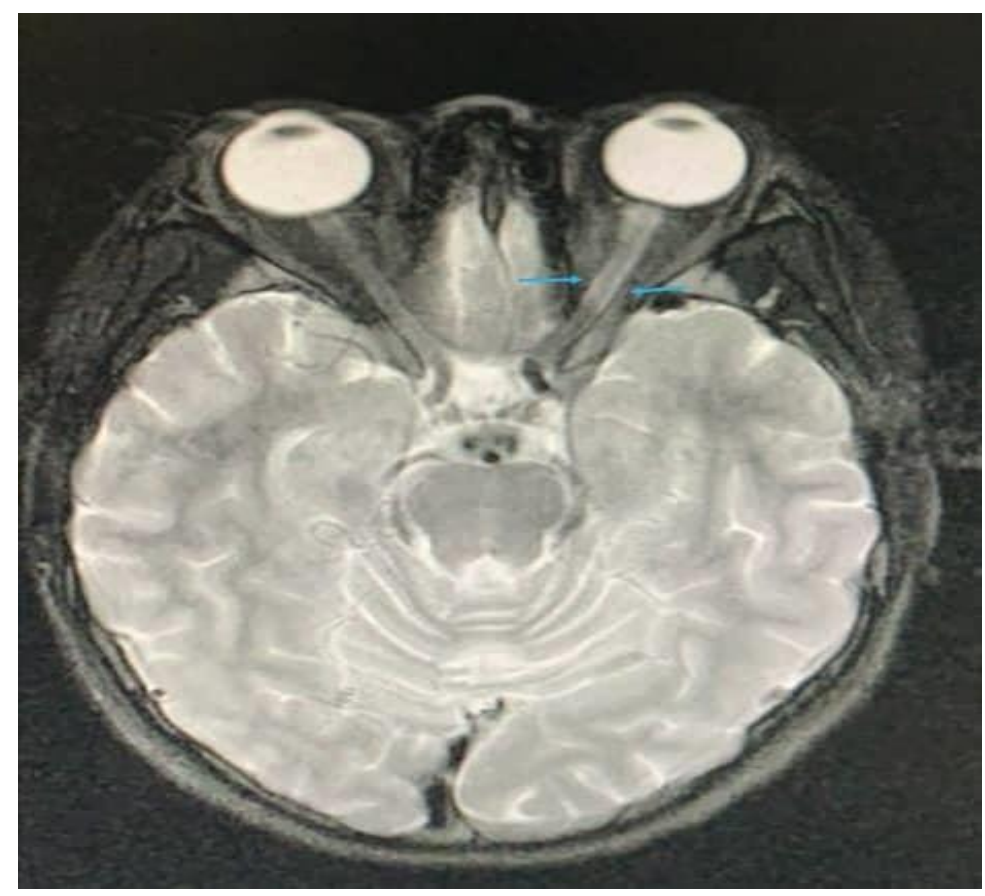

Figure 2: MRI showed the presence of 'tram track 'lesion over the left optic nerve

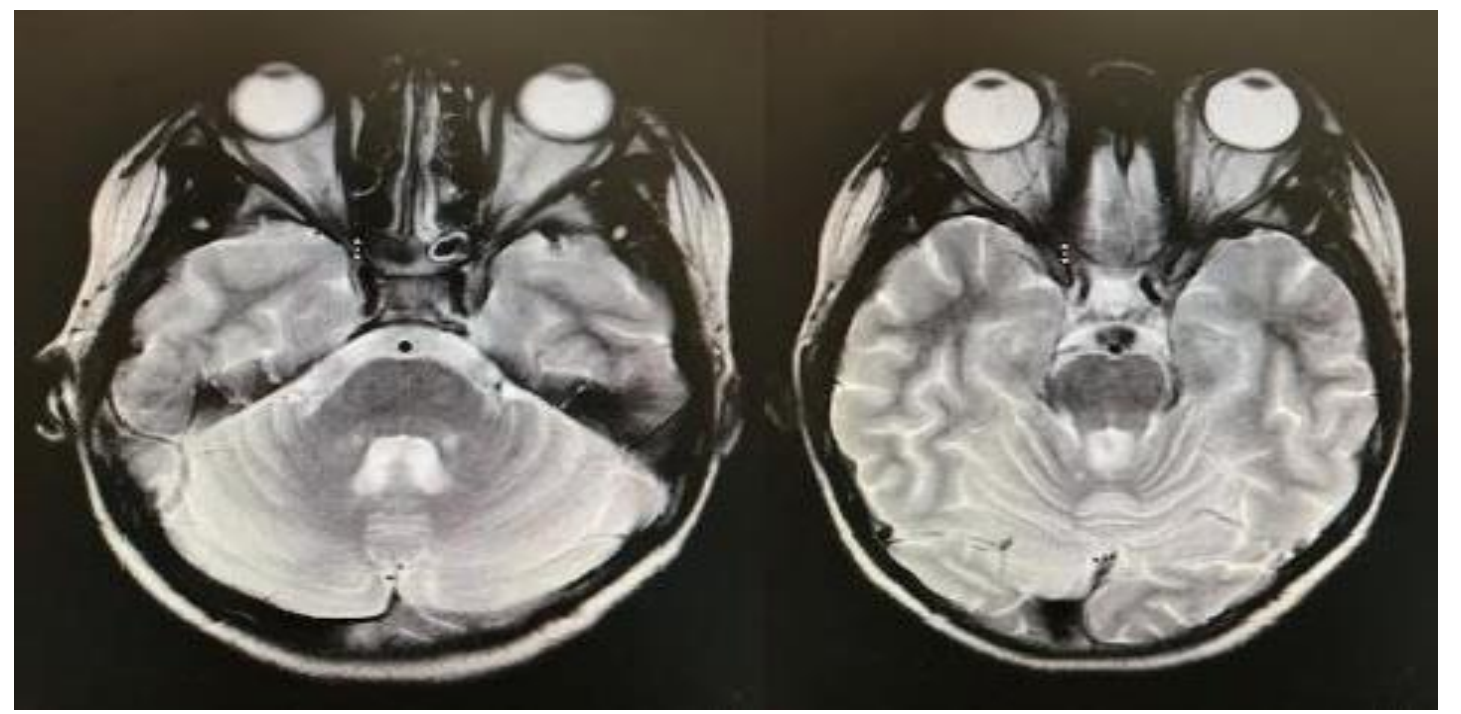

Figure 3: MRI showed the presence of inflammatory mass at the region of the left cavernous sinus extending till the orbital apex. 


\section{Discussion}

The prevalence of Tolosa Hunt Syndrome is about 1 case per million [3]. The pathophysiology shows the involvement of fibroblastic, lymphocytic, and plasmocytic infiltration of cavernous sinus which may extend to superior orbital fissure and may involve optic nerve [7]. Dr. Eduardo Tolosa described the pathophysiology as "nonspecific, chronic inflammation of the septa and wall of the cavernous sinus with the proliferation of fibroblasts an infiltration with lymphocytes and plasma cells." [1]. Tolosa Hunt syndrome with cerebral involvement and optic perineuritis is a rare entity. One case reported an association of Tolosa hunt syndrome with cerebral infarction, with involvement of carotid stenosis [5]. However, in this case, there are no abnormalities of the carotid artery. Blood investigation was taken to rule APLS, but all showed normal findings. Lumbar puncture was done and noted pressure is normal and CSF results are all normal. It is postulated that the mechanism of cerebral infarction in this patient might be due to hemodynamically changes that occurs which result in ischemia and infarction in the cerebral pontine. About this presentation, TSH should be recognized as a potentially reversible cause of stroke.

Another assumption that contributes to this presentation might be due to multiple sclerosis, since Tolosa Hunt syndrome rarely occurs as recurrent, in which this patient came back as recurrent after 2 months of completing her steroid therapy. However other symptoms of multiple sclerosis such as weakness of limb, tingling pain in parts of the limb, slurred speech, and other neurological symptoms are absent.

\section{Conclusion}

THS with cerebral involvement and optic perineuritis is a rare entity. Involvement of THS and cerebral infarction is uncommon. Contrasted MRI is a diagnostic modality of choice. The early course of steroid

\section{References}

1. Sci-Hub | PERIARTERITIC LESIONS OF THE CAROTID SIPHON WITH THE CLINICAL FEATURES OF A CAROTID INFRACLINOIDAL ANEURYSM. Journal of Neurology, Neurosurgery \& Psychiatry, 17(4), 300302. 10.1136/jnnp.17.4.300. Accessed June 7, 2021. https://scihub.do/http://dx.doi.org/10.1136/jnnp.17.4.300\#

2. Sci-Hub | Painful Ophthalmoplegia*. American Journal of Ophthalmology, 61(6), 1466-1472. 10.1016/00029394(66)90487-9. Accessed June 20, 2021.https://scihub.do/10.1016/0002-9394(66)90487-9

3. Iaconetta G, Stella L, Esposito M, Cappabianca P. TolosaHunt Syndrome Extending in the Cerebellopontine Angle: Cephalalgia. Published online June 26, 2016. Accessed June 7, 2021. https://journals.sagepub.com/doi/10.1111/j.14682982.2005.00924.x

4. Mendonca TM, Achappa B, Kamath SJ, Rodrigues GR. Optic
Further investigation including MRI spine and lumbar puncture might need to be carried out to rule out multiple sclerosis in this patient. Contrasted MRI is the diagnostic imaging for this condition. OnMRI presence of asymmetrical enlargement of the left cavernous sinus, orbital apex and adjacent dural thickening pointed toward Tolosa hunt syndrome. Also noted thickening of the left optic nerve with streakiness seen surrounding the left optic nerve which suggestive of left perineuritis.

Most of the reported cases successfully treated with intravenous methylprednisolone and showed favorable outcomes. One case report found an association of Tolosa hunt syndrome and optic perineuritis which resolved with intravenous methylprednisolone 1g/day for 5 days followed by oral prednisolone $1 \mathrm{~g} / \mathrm{kg}$ body weight with slow tapering [4]. Another case found of Tolosa Hunt Syndrome with underlying latent tuberculosis, treated also with IV methylprednisolone $1 \mathrm{~g}$ OD administered for 5 days as reported by Rafique Ali et al [6]. For this case on the first presentation, the symptoms in this patient resolve spontaneously without steroid. However, the patient came back with a similar presentation 1 week after discharge, and symptoms improved with iv methylprednisolone $1 \mathrm{~g} /$ day given for 5 days followed by oral prednisolone $1 \mathrm{~g} / \mathrm{kg}$ body weight with slow tapering. Since both Tolosa hunt syndrome and optic perineuritis are inflammatory disorders, an early course of steroid commencement may hasten the recovery as well as prevent progression complications.

commencement may hasten the recovery as well as to prevent progression and complication.

Perineuritis in a Case of Tolosa-Hunt Syndrome. J Neurosci Rural Pract. 2019;10(3):504-505. doi:10.1055/s-00391697245

5. Triplett JD, Chemmanam T. Tolosa-hunt syndrome associated severe internal carotid artery stenosis resulting in cerebral infarcts. J Neurol Neurosurg Psychiatry. 2017;88(5):e1-e1. doi:10.1136/jnnp-2017-316074.58

6. Aa RA, T I, J Y, N R. Tolosa-Hunt Syndrome with underlying latent tuberculosis: a diagnostic dilemma. Int J Ophthalmol. 2021;14(4):633-635. doi:10.18240/ijo.2021.04.24

7. Lachanas VA, Karatzias GT, Tsitiridis I, Panaras I, Sandris VG. Tolosa-Hunt syndrome misdiagnosed as sinusitis complication. J Laryngol Otol. 2008;122(1):97-99. doi:10.1017/S0022215106005317 